\title{
AncestralClust: Clustering of Divergent Nucleotide Sequences by Ancestral Sequence Reconstruction using Phylogenetic Trees
}

\author{
Lenore Pipes ${ }^{1, *}$ and Rasmus Nielsen ${ }^{1,2,3 *}$ \\ ${ }^{1}$ Department of Integrative Biology, University of California-Berkeley, Berkeley, 94707, USA, \\ ${ }^{2}$ Department of Statistics, University of California-Berkeley, Berkeley, CA 94707, USA, and \\ ${ }^{3}$ Globe Institute, University of Copenhagen, 1350 København K, Denmark \\ *To whom correspondence should be addressed.
}

\begin{abstract}
Motivation: Clustering is a fundamental task in the analysis of nucleotide sequences. Despite the exponential increase in the size of sequence databases of homologous genes, few methods exist to cluster divergent sequences. Traditional clustering methods have mostly focused on optimizing high speed clustering of highly similar sequences. We develop a phylogenetic clustering method which infers ancestral sequences for a set of initial clusters and then uses a greedy algorithm to cluster sequences.

Results: We describe a clustering program AncestralClust, which is developed for clustering divergent sequences. We compare this method with other state-of-the-art clustering methods using datasets of homologous sequences from different species. We show that, in divergent datasets, AncestralClust has higher accuracy and more even cluster sizes than current popular methods.
\end{abstract}

Availability and implementation: AncestralClust is an Open Source program available at https://github.com/lpipes/ancestralclust.

Contact: Ipipes@berkeley.edu

Supplementary information: Supplementary figures and table are available online.

\section{Introduction}

Traditional clustering methods such as UCLUST (Edgar, 2010) and CD-HIT (Fu et al., 2012) use hierarchical or greedy algorithms that rely on user input of a sequence identity threshold. These methods were developed for high speed clustering of a vast quantity of highly similar sequences (Ghodsi et al., 2011; Li et al., 2001; Edgar, 2010) and, generally, these methods are considered unreliable for identity thresholds $<75 \%$ because of either the poor quality of alignments at low identities (Zou et al., 2018) or because the performance of the method drops dramatically with low identities (Huang et al., 2010). At low identities, these methods produce uneven clusters where the majority of sequences are contained in only one or a few clusters (Chen et al., 2018). A high variance in cluster sizes may reduce the utility of clustering for many practical purposes since the goal of clustering typically is to reduce computational complexity of downstream analyses that are limited by the size of the largest clusters. Clustering of divergent sequences is an important step in genomics analysis because it allows for an early divide-and-conquer strategy that will significantly increase the speed of downstream analyses (Zheng et al., 2018) and many fundamental questions in metagenomics can be addressed by clustering of divergent sequences, such as the identification of gene families, and identification of sequences at the order, class, or phylum taxonomic levels. Currently, there are no clustering methods that can accurately cluster large taxonomically divergent metabarcoding reference databases such as the Barcode of Life database (Ratnasingham and Hebert, 2007) in relatively even clusters. Only a few other methods, such as SpClust (Matar et al., 2019) and TreeCluster (Balaban et al., 2019), exist for clustering potentially divergent sequences. SpClust creates clusters based on the use of Laplacian Eigenmaps and a Gaussian Mixture Model based on a similarity matrix calculated on all input sequences. While this approach is highly accurate, the calculation of an all-to-all similarity matrix is computationally demanding. 
For example, an all-by-all comparison for clustering 8 million environmental DNA (eDNA) reads by Rusch et al. (2007) took $>1$ year on a 100-CPU cluster. TreeCluster uses user-specified constraints for splitting a phylogenetic tree into clusters. However, TreeCluster requires an input tree and even though some phylogenetic methods exist to estimate trees from a large number of sequences (Stamatakis, 2014), it can also be prohibitively slow for large numbers of divergent sequences where a phylogenetic tree is difficult to estimate reliably. With the increasing size of reference databases (Schoch et al., 2020), there is a need for new computationally efficient methods that can cluster divergent sequences. Here we present AncestralClust which is specifically developed for clustering of divergent metabarcoding reference sequences in clusters of relatively even size.

\section{Methods}

To cluster divergent sequences, we developed AncestralClust written in $\mathrm{C}$ (Figure 1). The algorithm proceeds by first (1) selecting $r$ random sequences for pairwise alignment using the wavefront algorithm (Marco-Sola et al., 2020). We choose a random subset of sequences to reduce the computational burden of performing all-to-all alignments. The wavefront algorithm, which is an approximating algorithm for pairwise alignment with affine gap penalty, is used as a trade-off between computational time and accuracy since it runs in time linear with the sequence length and divergence. If computational time is not a concern the NeedlemanWunsch algorithm (Needleman and Wunsch, 1970), which has computational complexity that is quadratic in sequence length, can also be used for alignments. (2) A Jukes-Cantor (Jukes et al., 1969) distance matrix is then constructed from the alignments and a Neighbor-joining phylogenetic tree (Saitou and Nei, 1987) is estimated. The Jukes-Cantor model is chosen for computational speed, but more complex models could in principle be used to potentially increase accuracy but also increase computational time. (3) The leaves are clustered by cutting the $b-1$ longest branches in the tree to yield $b$ subtrees (corresponding to $b$ clusters of leaf nodes). These subtrees comprise the initial starting clusters. The next step (4) is to estimate the ancestral sequence in the root of each subtree. To increase accuracy, the sequences in each initial starting cluster are re-aligned in a multiple sequence alignment using kalign3 (Lassmann, 2020). Kalign3 was chosen to perform the multiple sequence alignments because of its accuracy and speed. Next, a new Neighbor-joining tree (Saitou and Nei, 1987) is constructed from each initial starting cluster, and each tree is midpoint rooted (Farris, 1972). The midpoint root method was chosen for computational speed. The ancestral sequences at the root of the tree of each cluster are estimated using the maximum of the posterior probability of each nucleotide using standard programming algorithms from phylogenetics (see e.g., Yang, 2014). The ancestral sequences are used as the representative sequence for each cluster. Next, (5) the remaining sequences (the ones currently not assigned to any cluster) are aligned to each of the $b$ ancestral sequences and a Jukes-Cantor distance is calculated. If the shortest distance to any of the $b$ ancestral sequences is larger than the average distance between clusters, the sequence is saved for assignment using an iterative algorithm where the sequence will be contained in a new cluster. If the shortest distance to any of the $b$ ancestral sequences is less than or equal to the average distance between clusters, the sequence is assigned to each cluster based on the shortest Jukes-Cantor distance from the wavefront algorithm alignment between the sequence and the $b$ ancestral sequences. This iterative algorithm proceeds by repeating steps 1 to 5 above for the previously unassigned sequences. If the number of sequences is $<r$, then $r$ becomes the number of unassigned sequences. In each iteration after the first iteration, a cut of a branch in the phylogenetic tree is chosen if the branch is longer than the average length of branches cut in the first iteration. We iterate this process until all sequences are assigned to a cluster.

Algorithm 1 provides an overview of the algorithm using the following notation: $N$ is the number of sequences to assign, $A=$ $\left\{A_{1}, \ldots, A_{N}\right\}$ is the set of sequences, $B=\left\{B_{1}, \ldots, B_{r}\right\}$ is a set of $r$ randomly chosen sequences, $\Omega=\left\{\omega_{1}, \ldots, \omega_{k}\right\}$ is the set of all $k$ clusters to be returned by the algorithm, i.e. a partition of $A$ into $k$ sets, $D$ is a matrix of Jukes-Cantor distances, Tree is a binary tree, $E=\left\{E_{1}, \ldots, E_{b}\right\}$ is a partition of sequences, $q$ is the average length of branches cut in the first iteration, $\gamma$ is a midpoint rooting (a point in the tree), $X_{i}$ is the ancestral sequence reconstruction at $\gamma_{i}$ for Tree $_{i}$ estimated from the sequences in $E_{i}$, and $\nu$ is the average distance between clusters. 


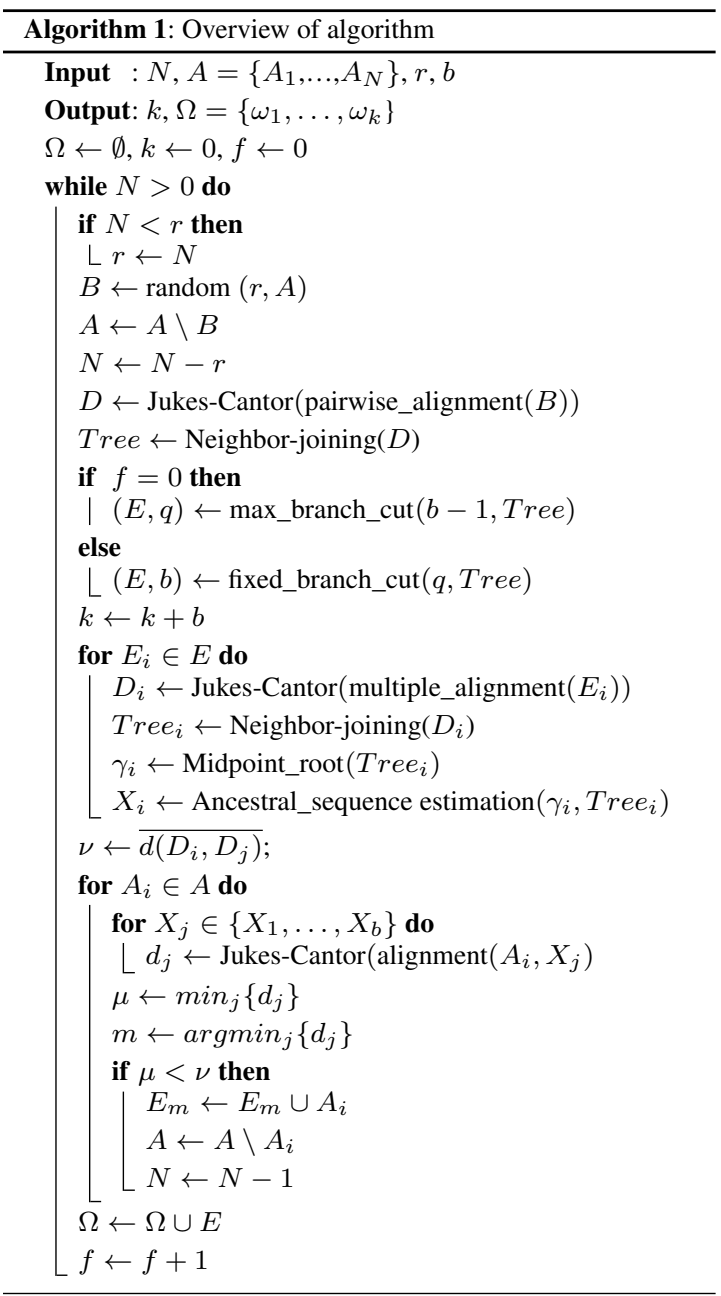

Here $B \leftarrow \operatorname{random}(r, A)$ is an operation in which $r$ distinct sequences are selected uniformly at random from $A$ to form the set of sequences $B$.

$(E, q) \leftarrow$ max_branch_cut $(b-1$, Tree $)$ is an operation in which the set of leaf nodes in Tree are divided into a partition $E$ with $b$ subsets, by cutting the $b-1$ longest branches in Tree, and in which $q$ is set to be equal to the average length of the branches cut.

$E \leftarrow$ fixed_branch_cut $(q$, Tree $)$ is an operation in which the set of leaf nodes in Tree are partitioned by cutting all branches in Tree with a length larger than $q$ to form a partition $E$ with $b$ subsets.

$d\left(D_{i}, D_{j}\right)$ is the average Jukes-Cantor distance between sequences in $E_{i}$ and $E_{j}$ and $\overline{d\left(D_{i}, D_{j}\right)}$ is the average of this quantity over all pairs $\left(E_{i}, E_{j}\right), i \neq j$, in $E$.

In praxis, only one or two iterations are needed for most data sets if $r$ is defined to be sufficiently large. The method is parallelized during the calculation of $D$, during the multiple sequence alignment (multiple_alignment $\left(E_{i}\right)$ ), and the assignment of unassigned sequences to clusters.
This procedure relies on arbitrary choices of $r$ and $b$. However, we calibrate the choices of $r$ and $b$ using a procedure described in Appendix A.

Notice that when aligning the $r$ sequences in $B$, a pairwise alignment is first used to define clusters and then a multiple alignment is used within each cluster when estimating phylogenetic trees. While this procedure does save computational time, as multiple alignments are expensive, the main reason for this two-step procedure is that multiple alignments, that include highly divergent sequences, can negatively affect alignment accuracy even among more similar sequences in the alignment. Initially, generating a large combined multiple alignment for all sequences in $B$ does, therefore, not lead to as good of a performance when estimating phylogenetic trees within each cluster, as when a multiple alignment is performed separately for each cluster.

We compare AncestralClust to two state-of-the-art clustering methods, UCLUST (Edgar, 2010) and CD-HIT (Fu et al., 2012), and a clustering method specifically developed for divergent sequences, SpClust (Matar et al., 2019). We use a variety of measurements to assess the accuracy and evennness of the clustering. We first calculate two traditional measures of accuracy: purity and normalized mutual information (NMI), used in Bonder et al. (2012). Although we acknowledge that inaccuracies in taxonomy exist in public sequence databases (see Nilsson et al. (2006)), for the purpose of evaluating performance, we define a taxonomic group as belonging to phylum, class, order, family, genus, or species as classified by the National Center for Biotechnology Information (NCBI) taxonomic system. Since taxonomic groups are not comparable across taxonomic levels (i.e., classes compared to genera), we calculate accuracy measures for each taxonomic level separately.

To describe the performance measures, we first need to introduce some notation. $w_{i}$ is, as previously defined, the set of sequences in cluster $i$. The number of different sequences in cluster $i$ is the cardinality of $w_{i},\left|w_{i}\right|$.

The purity of clusters, as defined by Manning (1988), is then calculated as:

$$
\operatorname{purity}(\Omega, C)=\frac{1}{N} \sum_{i=1}^{k} \max _{j}\left|\omega_{i} \cap c_{j}\right|
$$

where $C=\left\{c_{1}, c_{2}, \ldots, c_{d}\right\}$ is the partition of the data into $d$ taxonomic groups, where $c_{j}$ is the set of all sequences belonging to taxonomic group $j$, and $N$ is the total number of sequences. Notice that purity takes a value in $\{k / N,(k+1) / N, \ldots, 1\}$ and is equal to 1 if there are no clusters that contain more than one taxonomic group. Purity tends to increase as the number of clusters increases. For example, purity becomes 1 when each sequence is assigned to its own cluster.

We next describe the calculation of NMI. First, we define the proportion of sequences in cluster $i$ is $q_{i}=\left|\omega_{i}\right| / N$ and the entropy of the clusters as

$$
H(\Omega)=-\sum_{i=1}^{k} q_{i} \log _{2}\left(q_{i}\right) .
$$


The proportion of sequences with taxonomic assignment $j$ is $p_{j}=$ $\left|c_{j}\right| / N$. The entropy of the taxonomic groups is defined as

$$
H(C)=-\sum_{j=1}^{d} p_{j} \log _{2}\left(p_{j}\right)
$$

We also define the frequency of assignment $j$ in cluster $i$ as

$$
p_{j \mid i}=\left|\omega_{i} \cap c_{j}\right| /\left|\omega_{i}\right|,
$$

and the conditional entropy as

$$
H(C \mid \Omega)=-\sum_{i=1}^{k} q_{i} \sum_{j=1}^{d} p_{j \mid i} \log _{2}\left(p_{j \mid i}\right) .
$$

NMI is then calculated as

$$
N M I(\Omega, C)=\frac{2(H(C)-H(C \mid \Omega))}{[H(\Omega)+H(C)]}
$$

To measure the evenness of the clusters, we use the Coefficient of Variation, which is calculated as:

$$
C V=\frac{\sqrt{\sum_{j=1}^{d}\left(\left|c_{j}\right|-m\right)^{2} /(d-1)}}{m}
$$

where $m=\frac{\sum_{j=1}^{d}\left|c_{j}\right|}{d}$ is the mean size of the clusters. We also use a taxonomic incompatibility measure to assess the accuracy of the clusters. A taxonomic incompatibility is assigned if two taxonomic groups both exist in two different clusters. One taxonomic group can be split into multiple clusters, but if taxonomic groups are monophyletic, two groups should not both be found in more than one cluster. The total taxonomic incompatibility is then calculated by summing over all species found in the data set. More precisely, let $S_{\omega_{i}}\left(c_{l}, c_{j}\right)$ be an indicator variable that returns one if species $c_{l}$ and $c_{j}$ are both found in cluster $i$ (i.e., $\left.\left|\omega_{i} \cap c_{l}\right|\left|\omega_{i} \cap c_{j}\right|>0\right)$ and zero otherwise. Then the taxonomic incompatibility is defined as:

$$
T I(\Omega, C)=\sum_{l=1}^{d-1} \sum_{j=i+1}^{d} \max \left\{0, \sum_{i=1}^{k} S_{\omega_{i}}\left(c_{l}, c_{j}\right)-1\right\}
$$

Notice that this measure does not penalize paraphyletic clusters but only penalizes clustering that is strictly incompatible with a phylogentic tree, i.e. polyphyletic clusters.

All three measures (purity, NMI, and taxonomic incompatibility) are very sensitive to both the number of clusters and the variance in cluster size. For example, if all sequences are assigned to different clusters or if all sequences are assigned to the same cluster, the taxonomic incompatibility is, by definition, zero. In general, taxonomic incompatibility has the potential to be highest, and NMI has the potential to be lowest, when there is an intermediate amount of clusters of equal size. With high variance in cluster size there is less potential for generating clusters with high taxonomic incompatibility of low NMI. To address these issues and in order to allow fair comparison when numbers of clusters and variance in cluster sizes vary, we calculated the relative purity, relative NMI, and relative incompatibility. We calculate these measures by scaling them relative to their expected values under random assignments given the number of clusters and the cluster sizes. We estimate relative NMI by dividing the raw NMI score by the average NMI of 10 clusterings, in which sequences have been assigned at random with equal probability to clusters, such that the cluster sizes are the same as the cluster sizes produced in the original clustering. We use the same procedure to convert the purity measure into relative purity and the taxonomic incompatibility measure into relative incompatibility.

\section{Results}

To assess performance of these clustering methods on random samples of divergent nucleotide sequences, we used 100 random samples of 10,000 sequences from three metabarcode reference databases $(16,18 \mathrm{~S}$, and Cytochrome Oxidase I (COI)) from the CALeDNA project Curd et al. (2019). We were unable to perform clustering of these data sets using SpClust because the program did not complete in a feasible amount of time (we allowed for a week of computational time to complete the clusterings of 100 random samples for each method). In the main figures, we display the results against UCLUST, but because of the high number of clusters and high Coefficient of Variation of cluster sizes from CD-HIT (see Figures S8, S15 and S16), we display the CD-HIT results in the supplement. For CD-HIT, we used the lowest possible similarity threshold, which is $80 \%$, to attempt to create clusters of similar sizes to AncestralClust.

We used the COI database to explore the relationships between taxonomic incompatibility and the number of clusters (Figure S1), taxonomic incompatibility and the Coefficient of Variation (Figure S2), and taxonomic incompatibility and both the number of clusters and the Coefficient of Variation (Figure S3). Notice that taxonomic incompatibility increases as the number of clusters increases and taxonomic incompatiblity decreases as the Coefficient of Variation decreases. Also, the relationships between relative incompatibility and the number of clusters (Figure S4), relative incompatibility and the Coefficient of Variation (Figure S5), and relative incompatibility and both the number of clusters and the Coefficient of Variation (Figure S6) similarly show the same increasing or decreasing trends for species, genus, and family taxonomic levels. This shows that comparisons of methods cannot be done fairly without also referencing differences in number of clusters and variance in cluster sizes inferred by the different methods.

We compared AncestralClust against UCLUST using relative NMI and the Coefficient of Variation for species (Figure 2), genus, family, order, class, and phylum levels (Figure S7) for the $16 \mathrm{~S}, 18 \mathrm{~S}$, and COI metabarcoding data sets. We used $r=750$ random initial sequences, which is $7.5 \%$ of the total number of sequences in each sample, and $b=15$ initial clusters. The choice of $r$ and $b$ is described in Appendix A. Results for CD-HIT (Figure $\mathrm{S} 8$ ) show that CD-HIT creates hundreds of clusters for every 
barcode with a high Coefficient of Variation and tends to have a lower relative NMI than AncestralClust. Notice in Figure 2 that relative $N M I$ tends to be higher with a lower coefficient of variation for AncestralClust across all barcodes. This suggests, that for these divergent eDNA sequences, AncestralClust provides clusterings that are more even in size and that are more consistent with conventional taxonomic assignment. We also measured relative purity and relative incompatibility and Coefficient of Variation using AncestralClust, UCLUST, and CD-HIT for the same data sets under the same running conditions. Notice in Figures 3 and 4, AncestralClust tends to create balanced clusters with higher relative purities and lower relative taxonomic incompatibilities compared to UCLUST at all taxonomic levels. For relative incompatibility for metabarcode 16S (Figure S9), AncestralClust performs noticeably better than UCLUST at the species and genus levels but at the family, order, class, and phylum levels it has either the same or slightly more taxonomic incompatibility, but with substantially lower Coefficient of Variation of the cluster sizes. Also, at the species, genus, and family levels, there is a clear negative correlation between UCLUST relative incompatibility and Coefficient of Variation. This illustrates the observation that clusterings with a higher variance in cluster sizes tend to generate lower taxonomic incompatibility. For relative purity for $16 \mathrm{~S}$, AncestralClust has noticeably higher relative purities than UCLUST at every taxonomic level (Figure S10).

At the order, class, and phylum levels for metabarcode $18 \mathrm{~S}$ AncestralClust tends to have less relative incompatibility (Figure $\mathrm{S} 11$ ) but not at the species, genus, or family level. For relative purity of 18S, AncestralClust shows higher relative purities than UCLUST at the family, order, class and phylum levels but lower relative purities at the species and genus levels (Figure S12). The reason for this difference in relative performance between low and high taxonomic levels is that when $d$, the number of taxonomic groups, approaches $N$, the total number of sequences in a sample, the performance measured become increasingly sensitive to the value of $k$. As defined by Equation 1, when $d=N$, purity takes the value $k / N$. So at lower taxonomic levels with large values of $d$, methods that defines a high number of clusters (large value of $k$ ) will tend to have higher purity (Equation 1). The same effect is observed for taxonomic incompatibility (Equation 4). When $d=N$ taxonomic incompatibility is 0 . Thus, as $d$ approaches $N$, taxonomic incompatibility becomes increasingly sensitive to values of $k$. This sensitivity to $k$ is observed in the raw values of purity (Figure S13) and incompatibility (Figure S14) where the average number of species, genera, and families in a sample is high $(8369.1,5129.9$, and 2524.2, respectively), while the average number of phyla in a sample is low (50.4). $16 \mathrm{~S}$ and COI have fewer taxonomic groups than $18 \mathrm{~S}$ at every taxonomic level and thus are less sensitive to $k$.

For CD-HIT, relative purities of $16 \mathrm{~S}, 18 \mathrm{~S}$, and COI tends to be similar or lower than AncestralClust at every taxonomic level (Figure S15). Additionally, relative incompatibility of $16 \mathrm{~S}$ and $18 \mathrm{~S}$ tends to be similar or higher than AncestralClust at every taxonomic level (Figure S16). For COI, relative incompatibility tends to be higher or similar at species, genus, and family levels, but lower at order, class, and phylum levels, but with substantially higher Coefficient of Variation in cluster size.

Next, we analyzed two data sets with different properties: one data set of divergent species from the same gene and another data set of 6 paralogous genes from species of the same phylum. In the first data set, we expect the sequences to cluster according to species. In the second data set, we expect the sequences to cluster according to genes. The first data set contained 13,043 sequences from the COI CaleDNA database from 11 divergent species that were from 7 different phyla and 11 different classes and the second data set contained sequences from 6 different genes from taxonomically similar species. First, we compared all methods using 13,043 COI sequences from the 11 different species (Table 1). Ideally, we expect these sequences to form 11 different clusters, each including all the sequences from one species. We chose identity thresholds to enforce the expected number of clusters for each method. We were unable to form 11 clusters using CD-HIT because the program does not allow clustering of sequences with identity thresholds $<80 \%$ at default parameters. For SpClust, we used the three precision modes (fast, moderate, and maxPrecision) available for the method. In this analysis, AncestralClust achieved a perfect clustering (the raw purity was 1 and relative incompatibility was 0 ) and it had the second lowest memory usage, although it was the second slowest. CD-HIT also had a raw purity of 1 but formed more than twice the number of clusters than expected. UCLUST was one of the fastest methods and used the least amount of memory but had the second lowest relative purity with the third highest relative NMI values. SpClust only identified one cluster, with a computational time of $\sim 2$ days. In comparison, AncestralClust took $\sim 5$ minutes and UCLUST used $<1$ second.

Next, we analyzed 'genomic set 1' from Matar et al. (2019), which consists of 39 sequences from 6 homologous genes (FCER1G, S100A1, S100A6, S100A8, S100A12, and SH3BGRL3 in Table 2). We expect these sequences to form 6 clusters. We varied the identity thresholds for UCLUST using thresholds 0.4, 0.6, and 0.8. For CD-HIT, we used the lowest identity threshold available on default parameters which is 0.8 . Since this data set contained 6 different genes, we calculated relative NMI and relative purity using genes as the categories instead of taxonomy, and did not use taxonomic incompatibility as an accuracy measure. Only AncestralClust and UCLUST produced the expected number of clusters, and among the methods and parameters that created the expected number of clusters, AncestralClust had the highest relative purity value. AncestralClust was the second slowest method and had the highest memory requirements due to the wavefront algorithm alignment, which is $\mathcal{O}(n s)$ in running time and $\mathcal{O}\left(s^{2}\right)$ in memory requirements, where $n$ is the read length and $s$ is the alignment score. Since alignments were performed using 6 different genes that were longer than $1.5 \mathrm{~kb}$ (the average sequence length was 2,387.9bp and the longest sequence was 5,363bp), this resulted in high values of $n$ and $s$. SpClust 
had the highest relative NMI but lower relative purity than AncestralClust for all precision modes, however, it failed to produce the expected number of clusters and found fewer clusters with a higher Coefficient of Variation than AncestralClust, making the results difficult to compare.

We measured the time and memory requirements of AncestralClust using data sets containing 100,1,000,10,000, and 100,000 sequences from both the $16 \mathrm{~S}$ and the COI reference database for $1 \mathrm{CPU}$ and 8 CPUs (Figure S23). We created "low" divergence data sets by randomly choosing 1 sequence from the database and selecting all of its nearest sequences based on taxonomy information. We also created "high" divergence data sets which were created by randomly choosing sequences from the database that are from different phyla. We used the wavefront algorithm to investigate whether there was a substantial increase of memory requirements with the "high" divergence data set given that the alignment algorithm is quadratic with respect to the alignment score. Unsurprisingly, the "high" divergence data sets had the longest running time (Figure S23A) and consumed the most memory (Figure S23B). However, there were not substantially large differences in running times and memory usage between "high" and "low" divergent data sets. Additionally, the run time was significantly reduced by using more CPUs. The use of more CPUs also did not substantially increase the memory requirements.

\section{Conclusions}

We developed a phylogenetic-based clustering method, AncestralClust, specifically to cluster divergent metabarcode sequences. We performed a comparative study between AncestralClust and widely used clustering programs UCLUST and CD-HIT, and for divergent sequences, SpClust. UCLUST is substantially faster than AncestralClust and should be the preferred method if computational speed is the main concern (i.e., quick clustering of a large amount of raw sequencing reads from next generation sequencing technologies for error correction). However, AncestralClust tends to form clusters of more even size with lower relative taxonomic incompatibility and higher relative $N M I$ and relative purity than other methods, for the relatively divergent sequences analyzed here. We recommend the use of AncestralClust when sequences are divergent, especially if a relatively even clustering is also desirable, for example for various divide-andconquer approaches where computational speed of downstream analyses increases faster than linearly with cluster size.

\section{Funding}

This work used the Extreme Science and Engineering Discovery Environment (XSEDE) Bridges system at the Pittsburgh Supercomputing Center through allocation BIO180028 and was supported by NIH grant 1R01GM138634-01.

\section{References}

Balaban, M., Moshiri, N., Mai, U., Jia, X., and Mirarab, S. (2019) Treecluster: Clustering biological sequences using phylogenetic trees. PloS one, 14(8), $\mathrm{e} 0221068$.

Bonder, M. J., Abeln, S., Zaura, E., and Brandt, B. W. (2012). Comparing clustering and pre-processing in taxonomy analysis. Bioinformatics, 28(22), 2891-2897.

Chen, Q., Wan, Y., Zhang, X., Lei, Y., Zobel, J., and Verspoor, K. (2018) Comparative analysis of sequence clustering methods for deduplication of biological databases. J. Data and Information Quality, 9(3).

Curd, E. E., Gold, Z., Kandlikar, G. S., Gomer, J., Ogden, M., O'Connell, T., Pipes, L., Schweizer, T. M., Rabichow, L., Lin, M., et al. (2019). Anacapa toolkit: an environmental dna toolkit for processing multilocus metabarcode datasets. Methods in Ecology and Evolution, 10(9), 14691475.

Edgar, R. C. (2010). Search and clustering orders of magnitude faster than blast. Bioinformatics, 26(19), 2460-2461.

Farris, J. S. (1972). Estimating phylogenetic trees from distance matrices. The American Naturalist, 106(951), 645-668.

Fu, L., Niu, B., Zhu, Z., Wu, S., and Li, W. (2012). Cd-hit: accelerated for clustering the next-generation sequencing data. Bioinformatics, 28(23), 3150-3152.

Ghodsi, M., Liu, B., and Pop, M. (2011). Dnaclust: accurate and efficient clustering of phylogenetic marker genes. BMC bioinformatics, 12(1), $1-11$.

Huang, Y., Niu, B., Gao, Y., Fu, L., and Li, W. (2010). CD-HIT Suite: a web server for clustering and comparing biological sequences. Bioinformatics, 26(5), 680-682.

Jukes, T. H., Cantor, C. R., et al. (1969). Evolution of protein molecules. Mammalian protein metabolism, 3, 21-132.

Lassmann, T. (2020). Kalign 3: multiple sequence alignment of large datasets.

Li, W., Jaroszewski, L., and Godzik, A. (2001). Clustering of highly homologous sequences to reduce the size of large protein databases Bioinformatics, 17(3), 282-283.

Manning, C. (1988). I. Introduction. ISEAS Publishing.

Marco-Sola, S., Moure López, J. C., Moreto Planas, M., and Espinosa Morales, A. (2020). Fast gap-affine pairwise alignment using the wavefront algorithm. Bioinformatics, (btaa777), 1-8.

Matar, J., Khoury, H. E., Charr, J.-C., Guyeux, C., and Chrétien, S. (2019). Spclust: Towards a fast and reliable clustering for potentially divergent biological sequences. Computers in biology and medicine, 114, 103439.

Needleman, S. B. and Wunsch, C. D. (1970). A general method applicable to the search for similarities in the amino acid sequence of two proteins. Journal of molecular biology, 48(3), 443-453.

Nilsson, R. H., Ryberg, M., Kristiansson, E., Abarenkov, K., Larsson, K.H., and Kõljalg, U. (2006). Taxonomic reliability of dna sequences in public sequence databases: a fungal perspective. PloS one, 1(1), e59.

Ratnasingham, S. and Hebert, P. D. (2007). Bold: The barcode of life data system (http://www. barcodinglife. org). Molecular ecology notes, 7(3), 355-364.

Rusch, D. B., Halpern, A. L., Sutton, G., Heidelberg, K. B., Williamson, S., Yooseph, S., Wu, D., Eisen, J. A., Hoffman, J. M., Remington, K. et al. (2007). The sorcerer ii global ocean sampling expedition: northwest atlantic through eastern tropical pacific. PLoS Biol, 5(3), e77.

Saitou, N. and Nei, M. (1987). The neighbor-joining method: a new method for reconstructing phylogenetic trees. Molecular biology and evolution, 4(4), 406-425.

Schoch, C. L., Ciufo, S., Domrachev, M., Hotton, C. L., Kannan, S., Khovanskaya, R., Leipe, D., Mcveigh, R., O'Neill, K., Robbertse, B., et al. (2020). Ncbi taxonomy: a comprehensive update on curation, resources and tools. Database, $\mathbf{2 0 2 0}$.

Stamatakis, A. (2014). Raxml version 8: a tool for phylogenetic analysis and post-analysis of large phylogenies. Bioinformatics, 30(9), 13121313 . 
bioRxiv preprint doi: https://doi.org/10.1101/2021.01.08.426008; this version posted September 27, 2021. The copyright holder for this preprint (which was not certified by peer review) is the author/funder, who has granted bioRxiv a license to display the preprint in perpetuity. It is made available under aCC-BY 4.0 International license.

Yang, Z. (2014). Molecular evolution: a statistical approach. Oxford University Press.

Zheng, W., Mao, Q., Genco, R. J., Wactawski-Wende, J., Buck, M., Cai, Y., and Sun, Y. (2018). A parallel computational framework for ultra-largescale sequence clustering analysis. Bioinformatics, 35(3), 380-388.

Zou, Q., Lin, G., Jiang, X., Liu, X., and Zeng, X. (2018). Sequence clustering in bioinformatics: an empirical study. Briefings in Bioinformatics, 21(1), 1-10.

\section{Appendix A}

To optimize the choice of $r$ and $b$, we used 100 random samples of 10,000 sequences from the COI database from the CALeDNA project (Curd et al., 2019) and first chose the values of 15, 100, 250, 500, 750, and 1000 for $r$ and set $b$ to 15 . We calculated relative NMI, relative purity, and relative incompatibility as described in the Methods. Relative NMI (Figure S17) and relative purity (Figure S18) tend to increase with increasing values of $r$ for species, genus, and family taxonomic levels. Additionally, relative incompatibility (Figure S19) tends to decrease with increasing values of $r$ for species, genus, family, order, and class taxonomic levels. We chose the value of $r$ to be 750 since it maximized all three of the performance measures at species, genus, and family taxonomic levels. To optimize $b$, we set $r$ to 750 and chose values of $5,10,15,20,50$, and 80 for $b$ for the same dataset. We also calculated relative NMI (Figure S20), relative purity (Figure S21), and relative incompatibility (Figure S22). While the choice of $b$ on relative NMI (Figure S20) and relative purity (Figure S21) showed opposite effects, the choice of $b$ had little effect on relative incompatibility (Figure S22). Relative NMI tends to increase as $b$ becomes smaller but relative purity tends to decrease as $b$ becomes smaller. We chose the value of $b$ to be 15 which maximized relative incompatibility at the species, genus, and family taxonomic levels. While this procedure for choosing $r$ and $b$ is based on a specific data set of COI sequences, we do not observe great dependence of the performance on the exact values of $r$ and $b$ (see Results) and recommend them for use in analyses of other data sets as well in the absence of other information. 
bioRxiv preprint doi: https://doi.org/10.1101/2021.01.08.426008; this version posted September 27, 2021. The copyright holder for this preprint (which was not certified by peer review) is the author/funder, who has granted bioRxiv a license to display the preprint in perpetuity. It is made available under aCC-BY 4.0 International license.

(1) Choose $r$ random sequences for initial clusters

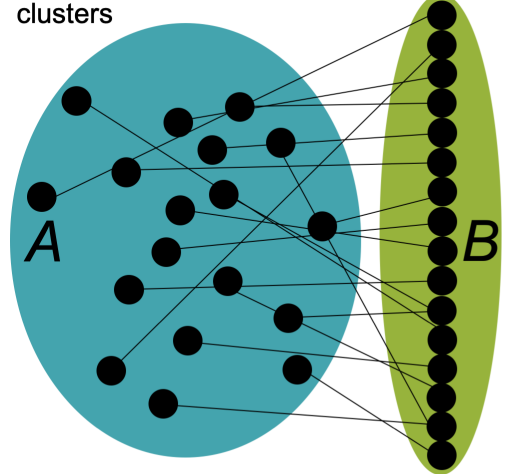

(2 and 3) Neighbor-joining tree estimation and $b-1$ cuts to yield $b$ initial clusters

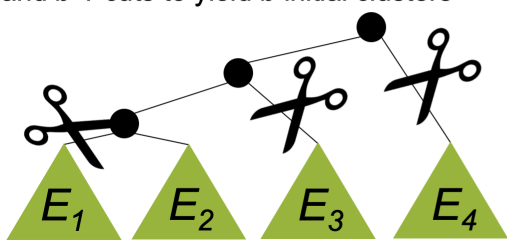

(4) Infer ancestral sequences for $b$ initial clusters $\begin{array}{cccc}\text { Tree }_{1} & \mathrm{Tree}_{2} & \mathrm{Tree}_{3} & \mathrm{Tree}_{4}\end{array}$

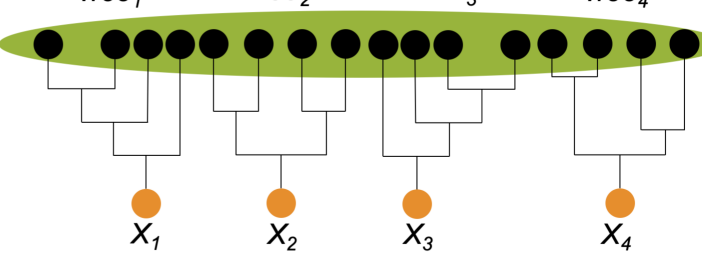

(5) Assign to a cluster using similarity to root sequence or save divergent sequences for next iteration

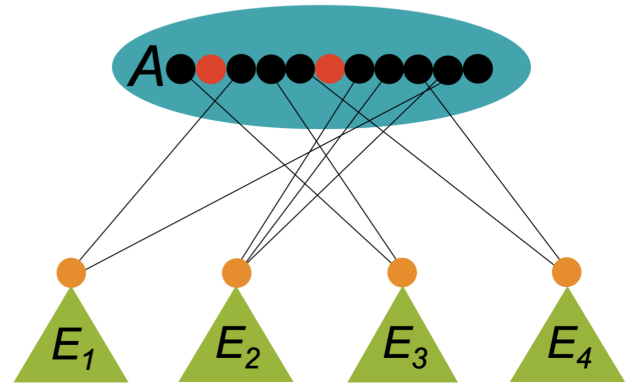

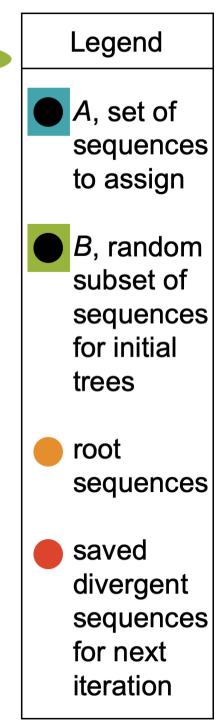

Figure 1. Overview of AncestralClust. In (1), $r$ random sequences are chosen from $A$ for the initial clusters. (2) Using the $r$ random sequences a Jukes-Cantor distance matrix is constructed. Using the distance matrix, a Neighbor-joining tree is estimated and in (3) $b-1$ cuts are made to create $b$ clusters. In (4), each cluster is aligned using a multiple sequence alignment and a Neighbor-joining tree is estimated. Each tree is midpoint rooted, and the ancestral sequences are reconstructed in the root node of each tree. In (5), the rest of the unassigned sequences in $A$, are then aligned to the ancestral sequences of each cluster and the shortest Jukes-Cantor distance to each ancestral sequence is calculated. If the shortest distance from the unassigned sequence to any of the ancestral sequences is larger than the average distance between clusters, then the unassigned sequence is saved for the next iteration. If the shortest distance to any of the ancestral sequences is less than or equal to the average distance between clusters, the sequence is assigned to the cluster with the shortest distance from its ancestral sequence. The process is iterated until all sequences are assigned to a cluster. In this specific example, $r=16$ and $b=4$.
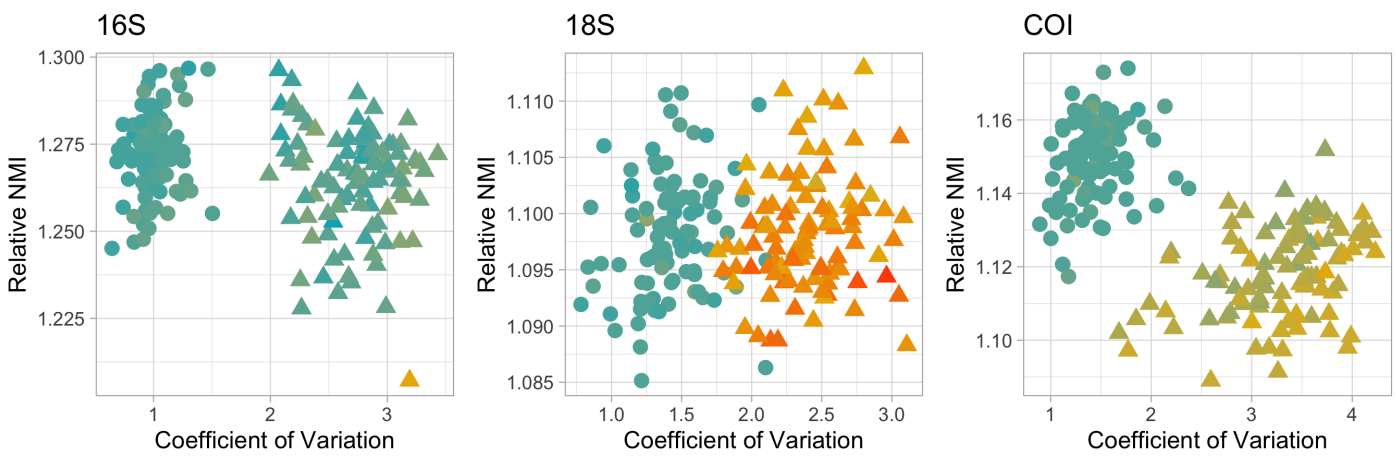

$\begin{array}{lllll}\text { Number of Clusters } & 20 & 30 & 40 & 50\end{array}$

Method AncestralClust

$\Delta$ UCLUST

Figure 2. Relative NMI at the species level against Coefficient of Variation for AncestralClust and UCLUST for 100 samples of 10,000 randomly chosen 16S, 18S, and COI reference sequences from the CALeDNA Project (Curd et al., 2019). The similarity threshold for UCLUST was 0.58. For AncestralClust, we used 750 initial random sequences with 15 initial clusters. 
bioRxiv preprint doi: https://doi.org/10.1101/2021.01.08.426008; this version posted September 27, 2021. The copyright holder for this preprint (which was not certified by peer review) is the author/funder, who has granted bioRxiv a license to display the preprint in perpetuity. It is made available under aCC-BY 4.0 International license.
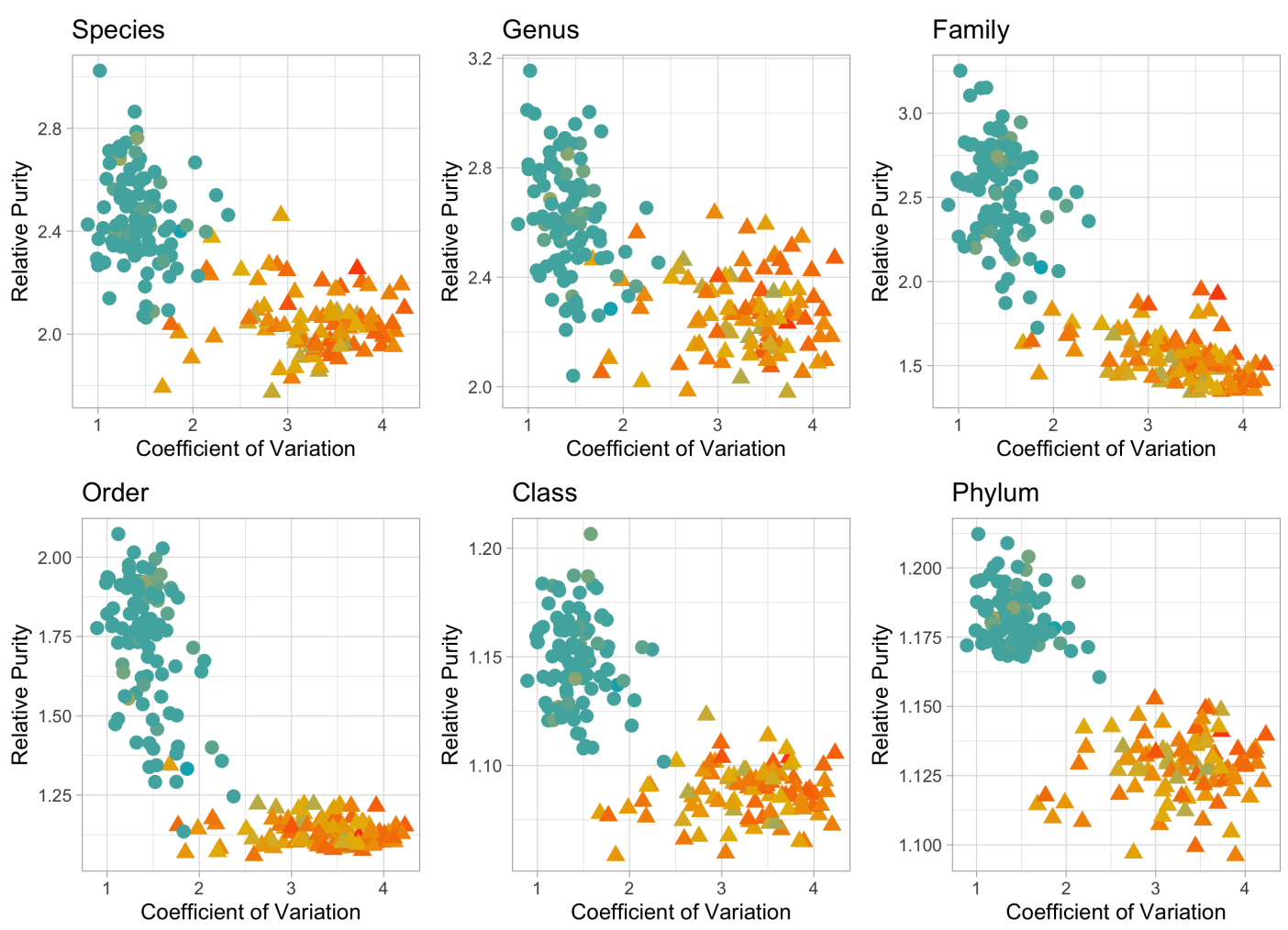

Number of Clusters $\begin{array}{rrrr}15 & 20 \quad 25 \quad 30 \quad 35\end{array}$

Figure 3. Relative purity against coefficient of variation for AncestralClust and UCLUST for 100 samples of 10,000 randomly chosen COI reference sequences. COI reference sequences are from the CALeDNA Project (Curd et al., 2019). The similarity threshold for UCLUST was 0.58. For AncestralClust, we used 750 initial random sequences with 15 initial clusters. 
bioRxiv preprint doi: https://doi.org/10.1101/2021.01.08.426008; this version posted September 27, 2021. The copyright holder for this preprint (which was not certified by peer review) is the author/funder, who has granted bioRxiv a license to display the preprint in perpetuity. It is made available under aCC-BY 4.0 International license.

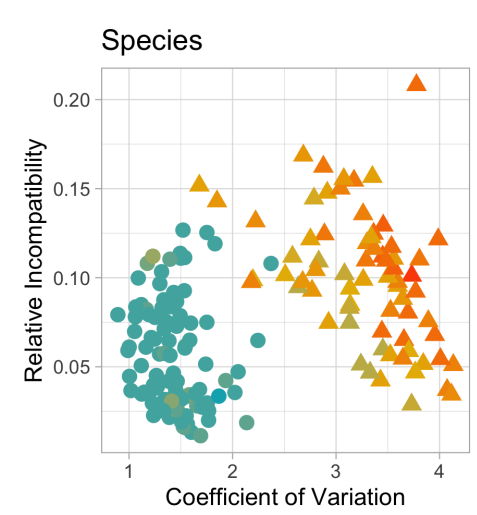

Coefficient of Variation

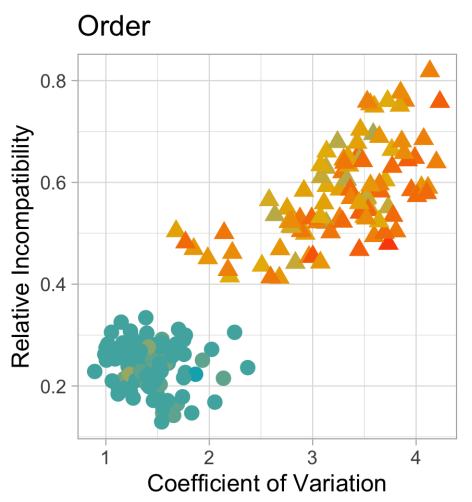

Number of Clusters
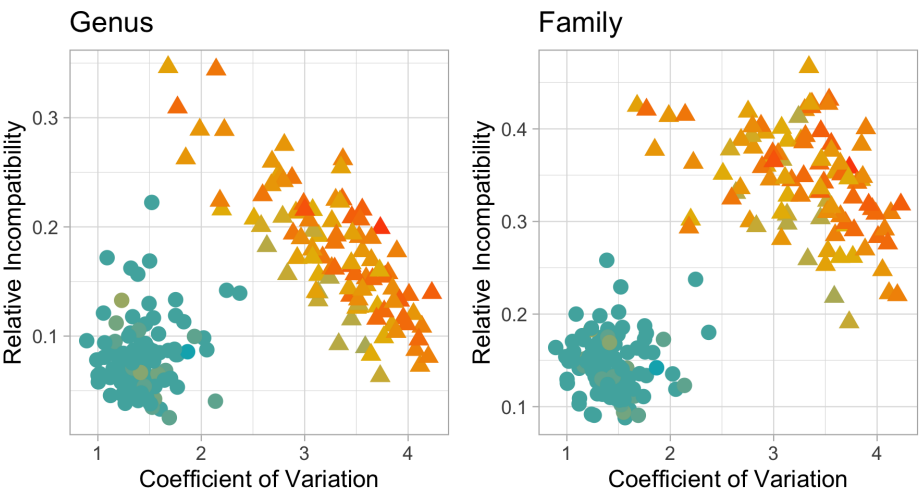

Class
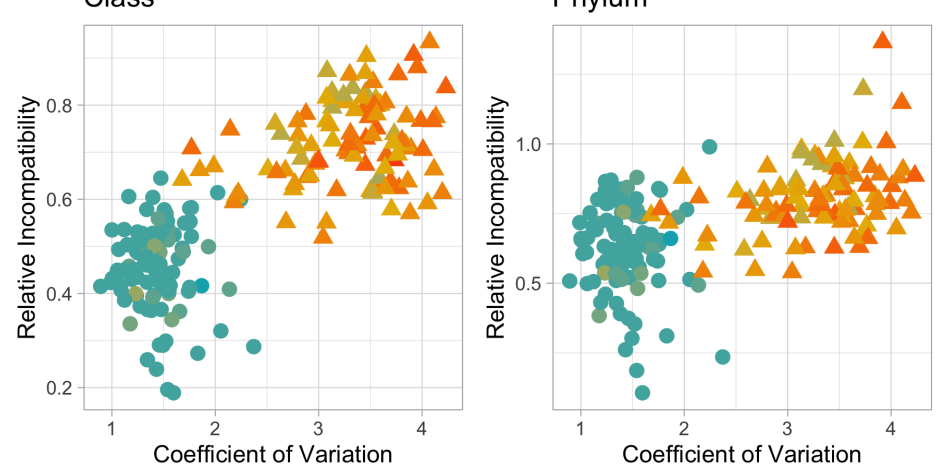

Method

AncestralClust

$\Delta$ UCLUST

Figure 4. Relative incompatibility against coefficient of variation for AncestralClust and UCLUST for 100 samples of 10,000 randomly chosen COI reference sequences. COI reference sequences are from the CALeDNA Project (Curd et al., 2019). The similarity threshold for UCLUST was 0.58. For AncestralClust, we used 750 initial random sequences with 15 initial clusters.

Table 1. Comparisons of clustering methods using 13,043 COI sequences from 11 different species. The list of species can be found in Table S1. Relative purity, relative incompatibility, and relative NMI were calculated at the taxonomic rank of species. For UCLUST, the identity thresholds were chosen to force the expected 11 number of clusters. For CD-HIT, the lowest possible identity was chosen which is 0.8 . In the case of SpClust, Coefficient of Variation cannot be calculated for 1 cluster. SpClust clusters were created with version 2.

\begin{tabular}{|c|c|c|c|c|c|c|c|}
\hline Method & $\begin{array}{c}\text { \# of } \\
\text { clusters }\end{array}$ & $\begin{array}{c}\text { Time } \\
\text { (sec) }\end{array}$ & $\begin{array}{c}\text { Mem } \\
\text { (MB) }\end{array}$ & $\begin{array}{c}\text { Relative } \\
\text { purity } \\
\text { (species) }\end{array}$ & $\begin{array}{c}\text { Relative } \\
\text { incompat. } \\
\text { (species) }\end{array}$ & $\begin{array}{c}\text { Relative } \\
\text { NMI } \\
\text { (species) }\end{array}$ & $\begin{array}{c}\text { Coeff. } \\
\text { of } \\
\text { Var. }\end{array}$ \\
\hline AncestralClust & 11 & 293.2 & 19.3 & 3.6271 & 0 & 551.09 & 0.8574 \\
\hline UCLUST & 11 & $<1$ & 9.9 & 3.1204 & 0.0182 & 474.63 & 0.8300 \\
\hline CD-HIT & 24 & 5.86 & 43.9 & 3.6180 & 0 & 241.66 & 1.2031 \\
\hline $\begin{array}{c}\text { SpClust } \\
\text { (fast) }\end{array}$ & 1 & 152046.5 & 2678.9 & 1 & 0 & 1 & - \\
\hline $\begin{array}{c}\text { SpClust } \\
\text { (moderate) }\end{array}$ & 1 & 188172.9 & 6457.6 & 1 & 0 & 1 & - \\
\hline $\begin{array}{c}\text { SpClust } \\
\text { (maxPrecision) }\end{array}$ & 1 & 189577.1 & 6452.5 & 1 & 0 & 1 & - \\
\hline
\end{tabular}


bioRxiv preprint doi: https://doi.org/10.1101/2021.01.08.426008; this version posted September 27, 2021. The copyright holder for this preprint (which was not certified by peer review) is the author/funder, who has granted bioRxiv a license to display the preprint in perpetuity. It is made available under aCC-BY 4.0 International license.

Table 2. Comparisons of clustering methods using 39 sequences from 6 paralogous genes from Matar et al. (2019). 'id' refers to the identity threshold used. We used identity thresholds of $0.4,0.6$, and 0.8 for UCLUST. We used precision levels of fast, moderate, and maximum for SpClust using version 1 since version 2 only produced 1 cluster for all modes.

\begin{tabular}{|c|c|c|c|c|c|c|}
\hline Method & $\begin{array}{c}\text { \# of } \\
\text { clusters }\end{array}$ & $\begin{array}{l}\text { Time } \\
\text { (sec) }\end{array}$ & $\begin{array}{c}\text { Memory } \\
\text { (MB) }\end{array}$ & $\begin{array}{c}\text { Relative } \\
\text { Purity }\end{array}$ & $\begin{array}{c}\text { Relative } \\
\text { NMI }\end{array}$ & $\begin{array}{c}\text { Coeff. } \\
\text { of } \\
\text { Var. }\end{array}$ \\
\hline AncestralClust & 6 & 370.3 & 412.0 & 1.7619 & 1.8660 & 0.3982 \\
\hline $\begin{array}{l}\text { UCLUST } \\
(\mathrm{id}=0.4)\end{array}$ & 6 & 1 & 15.4 & 1.3810 & 1.5667 & 0.5396 \\
\hline $\begin{array}{l}\text { UCLUST } \\
(\mathrm{id}=0.6)\end{array}$ & 19 & 1 & 20.1 & 2.1538 & 1.4379 & 0.7166 \\
\hline $\begin{array}{l}\text { UCLUST } \\
(\mathrm{id}=0.8)\end{array}$ & 29 & 1.9 & 20.4 & 1.6923 & 1.1717 & 0.4565 \\
\hline $\begin{array}{l}\text { CD-HIT } \\
(\mathrm{id}=0.8)\end{array}$ & 31 & 0.48 & 39.9 & 1.2308 & 1.0950 & 0.4574 \\
\hline $\begin{array}{l}\text { SpClust } \\
\text { (fast) }\end{array}$ & 4 & 44.6 & 166.2 & 1.4167 & 2.2463 & 0.8432 \\
\hline $\begin{array}{c}\text { SpClust } \\
\text { (moderate) }\end{array}$ & 4 & 112.5 & 166.1 & 1.6818 & 2.4335 & 0.6453 \\
\hline $\begin{array}{l}\text { SpClust } \\
\quad(\max \\
\text { precision) }\end{array}$ & 4 & 570.1 & 166.0 & 1.6818 & 2.9449 & 0.6809 \\
\hline
\end{tabular}

